\title{
Metal-insulator transition and magnetic ordering in Hubbard models near the Nagaoka limit
}

\author{
Q. P. Li and Robert Joynt \\ Department of Physics and Applied Superconductivity Center \\ University of Wisconsin-Madison \\ 1150 University Avenue, Madison, Wisconsin 53706
}

(July 17, 2018)

\begin{abstract}
We study the metal-insulator transition and magnetic ordering in the Hubbard model using the particle-hole mapping. The analysis simplifies near the ferromagnetic limit. We find that the two dimensional(2D) Hubbard model has a charge excitation gap at half-filling for any finite $U$ in this region on both the bipartite square lattice and the nonbipartite triangular lattice. In some cases, the system goes through a first-order phase transition to become a paramagnetic metal as $S_{z}$ is lowered. We also discuss the extension to the doped case. We find that in the large U limit, a single doped hole has a bandwidth of order of J rather than t at $S_{z}=0$.
\end{abstract}

PACS numbers: 71.30.+h, 75.10.Jm, 05.30.Fk

Typeset using REVTEX 


\section{INTRODUCTION}

The Hubbard model is one of the most extensively studied strongly correlated Fermion models in condensed matter physics. 1ㅡㄴ Despite its simple appearance, it has been used to explain a wide variety of physical phenomena such as ferromagnetism, [5] antiferromagnetism, [6] metal-insulator transition, [1, 3] and more recently, it has been proposed as a model for high- $T_{c}$ superconductivity. [7] One of the interesting features of the Hubbard model is the possibility of transforming from the repulsive $(U>0)$ to the attractive $(U<0)$ model. It has been known for a long time that a positive-U Hubbard model can be mapped into a negative- $U$ model by making a particle-hole transformation on one of the spin species. [8 10] Here we use this mapping to study the magnetic and transport properties of Hubbard model on various lattices. One of the motivations to use this transformation is to make use of the existing knowledge about the attractive Fermion gas in finding a good trial wavefunction for the Hubbard model. For example, Leggett [11] and Nozieres and Schmitt-Rink [12] have shown that the BCS wavefunction contains the right physics of attractive Fermion gas in both the weak and the strong coupling limits, and it may be used as an interpolation scheme in between to describe the progressive buildup of pairing correlations in the ground state. Thus we can use a unified method for all U. Furthermore, this wavefunction (in the transformed variables) provides a natural description of the binding and unbinding of particle-hole-pairs. This binding can be regarded, in certain situations, as the Mott transition into the insulating state.

Because of the importance of Hubbard model, exactly soluble limits or modifications are of great interest, as evidenced by much work on the Nagaoka limit of small doping and large $\mathrm{U}$, and on the one-dimensional t-J model. By mapping the positive- $\mathrm{U}$ Hubbard model into a negative- $\mathrm{U}$ model, we are able to solve the Hubbard model in the limit of high total $\mathrm{z}$ spin, $S_{z}$ for all U. We find that dimensionality plays an important role in the metal-insulator transition in this limit. In1D and 2D, the Hubbard model always has a charge excitation gap at half-filling at high $S_{z}$, and therefore, is always an insulator for any finite $\mathrm{U}$ in the 
high- $S_{z}$ region. In three or higher dimensions, however, there exist a critical interaction $U_{b}$, which separates a metallic phase (small U) from an insulating phase (large U). Using the information from the exact solution, we construct a BCS type of many-body wavefunction for the lower $S_{z}$ case and calculate the phase diagram in the $S_{z}$ U plane. In our work, we choose to fix $S_{z}$ rather than apply an external magnetic field which acts on the spins. We discuss this choice in more detail below.

Using a particle-hole transformation for spin-up electrons, the Hubbard Hamiltonian

$$
H=-\sum_{i j \sigma} t_{i j} c_{i \sigma}^{\dagger} c_{j \sigma}+\text { h.c. }+U \sum_{i} c_{i \uparrow}^{\dagger} c_{i \uparrow} c_{i \downarrow}^{\dagger} c_{i \downarrow}
$$

can be written as 9

$$
H=\sum_{i j} t_{i j} h_{i}^{\dagger} h_{j}-\sum_{i j} t_{i j} d_{i}^{\dagger} d_{j}+\text { h.c. }+U \sum_{i} d_{i}^{\dagger} d_{i}-U \sum_{i} d_{i}^{\dagger} d_{i} h_{i}^{\dagger} h_{i}
$$

Here $h_{i}^{\dagger}=c_{i \uparrow}$ and $d_{i}^{\dagger}=c_{i \downarrow}^{\dagger}$ are the creation operators of the "hole" and the "doublon". $c_{i \sigma}^{\dagger}$ is the original electron creation operator. The "vacuum" state $\mid 0>$ has an up spin electron at every site. In this hole-doublon representation (referred as HDR hereafter), holes and doublons have an on-site attractive interaction $-U$, and doublons have a site energy $U$. The zero-radius bound state of a doublon and a hole at site $i$ is nothing but a spin down state at $i$. In the dilute limit, the bound state pair behaves like a hard-core boson. 12 Also note the change in sign of the hole kinetic energy, a crucial part of the transformation.

The rest of paper is organized as follows: In Sec. II, we present the exact solution in the high total spin limit. We show, by calculating the Kohn charge stiffness constant, that the system is an insulator when and only when there exists a bound state solution in HDR. We devote Sec. III to the lower total spin case. In Sec. IV, we present a possible generalization of our many-body wavefunction at the half-filling to the hole-doped case and use it to calculate the minimum energy and the bandwidth of the doped hole. We summarize our results in Sec. V. 


\section{THE HIGH TOTAL SPIN LIMIT}

Let us start with the exactly soluble problem of a single pair of doublon and hole, i.e., the band is half-filled and there is only one down spin, $S_{z}=(N-2) / 2$. Here $\mathrm{N}$ is the number of electrons. One of the motivation to study the single pair problem is that in $2 \mathrm{D}$, the existence of the bound state solution is the necessary and sufficient condition of the BCS many-body instability, [13] which in our case corresponds to a spin excitation gap at lower $S_{z}$, although the actual situation is more complicated because of the particle-hole mapping, as we shall discuss later in Sec. III.

The system consists of a single hole and a single doublon, all other sites being occupied by an up spin. In this background, both particles move freely, except for their attraction for one another, which is zero range. This two particle problem differs from the text book bound state problem only in that the particles move in a band of finite width and that the hole prefers to sit at the top, not the bottom, of this band. The latter circumstance implies that the lowest energy state of a bound pair may not have zero total momentum.

The wavefunction of the system consisting of one hole and one doublon with total momentum $\vec{K}$ in the HDR can be written as,

$$
\Psi_{\vec{K}}=\sum_{\vec{k}} \alpha_{\vec{k}} d_{\vec{k}}^{\dagger} h_{\vec{K}-\vec{k}}^{\dagger} \mid 0>
$$

Substituting Eqs.(2) and (3) into the Schrodinger equation yields,

$$
\alpha_{\vec{k}}=-\frac{A U / N}{E_{\vec{K}}-\epsilon_{\vec{q}}+\epsilon_{\vec{K}-\vec{q}}} .
$$

Here $\epsilon_{k}=-\sum_{j} t_{i j} e^{i \vec{k} \cdot \vec{r}_{j i}}$ is the dispersion relation for the non-interaction case and $\mathrm{A}$ is a normalization constant which cancels out in the eigenvalue equation. The eigenenergy of the system is given by

$$
\frac{1}{N} \sum_{\vec{q}} \frac{1}{E_{\vec{K}}-\epsilon_{\vec{q}}+\epsilon_{\vec{K}-\vec{q}}}=-\frac{1}{U} .
$$

We first consider the most simple case that $t_{i j}$ is only nonzero for nearest neighbor hopping on a d-dimensional hyper-cubic lattice, then $\epsilon_{k}=-2 t \sum_{m=1}^{d} \cos _{m}$. (We assume 
the lattice constant $a=1$ throughout this paper.) The doublon kinetic energy is minimum at $\vec{k}=0$, and the hole kinetic energy at $\vec{k}=(\pi, \pi, \ldots, \pi)$. For every given $\vec{K}$, one can calculate $E_{\vec{K}}$ from Eq.(5). We find that the minimum value of $E_{K}$ is reached at $K_{1}=K_{2}=$ $\ldots=K_{d}=\pi$, consistent with the kinetic energies of the particles. So Eq. (5) can be written as

$$
f(E)=\int \frac{d^{d} q}{(2 \pi)^{d}} \frac{1}{E+4 t \sum_{m=1}^{d} \cos q_{m}}=-\frac{1}{U}
$$

in the limit $N \rightarrow \infty$. Here $\mathrm{E}$ is the minimum value of $E_{\vec{K}}$ and the integration is over the first Brillouin zone. The structure of the solutions of Eq. (6) is well known. [14] There is an extended state continuum of eigenvalue E between energy $-4 t d$ and $4 t d$, and there may or may not be a bound state below the continuum lower edge $E_{-}=-4 t d$ depending on the interaction $\mathrm{U}$ and the dimensionality $\mathrm{d}$. For $\mathrm{d}=1$, the integral in Eq. (6) is divergent as $1 / \epsilon$ when E approaches $E_{-}$from below so that $f\left(E \rightarrow E_{-}\right) \rightarrow-\infty$. Also $f(E \rightarrow-\infty)=0$. This means that there is always a bound state solution no matter how small the interaction $\mathrm{U}$ is. $d=2$ is the marginal case, the integral in Eq. (6) is logarithmically divergent as E approaches $E_{-}$from below. So there is a bound state for any U, although the gap between the bound state and the extended state continuum decreases exponentially as $e^{-8 \pi t / U}$ when $\mathrm{U}$ is small. For $\mathrm{d}=3$, the integral of Eq. (6) is convergent as E approaches $E_{-}=-12 t$ and there exists a critical $U_{b}$ such that when $U<U_{b}$, there is no bound state. $U_{b}$ is given by

$$
\frac{1}{U_{b}}=\int \frac{d^{3} q}{(2 \pi)^{3}} \frac{1}{4 t\left(3-\sum_{m=1}^{3} \cos q_{m}\right)} .
$$

Numerical evaluation of the integral gives $U_{b} \simeq 7.916 t$. $U_{b}$ increases monotonically as $\mathrm{d}$ is increased past 3 .

Next we consider the single-pair hole-doublon problem on a 2D triangular lattice with the nearest neighbor hopping only. The sign difference of the hopping terms of doublons and holes due to the particle-hole transformation now becomes important. When the system is on a bipartite lattice, the minus sign can be simply "gauged" away by redefining $h_{i}^{\dagger}=s(i) c_{i \uparrow}$. Here $\mathrm{s}(\mathrm{i})$ equals 1 or -1 when the site i belongs to two different sublattices. After this 
redefinition, the new "hole" has the same hopping term as doublons and is able to hop coherently with the doublon which it forms a bound state with. Now the minimum of energy is reached when the transformed total momentum $\vec{K}=0$. When the Hubbard model is on a nonbipartite triangular lattice, however, the sign difference of the hopping terms of holes and doublons in Eq. (2) can not be gauged away, i.e., the hopping terms with $+t$ and $-t$ are not symmetric any more. For example, the minimum value of the doublon hopping term is $-6 t$ on a $2 \mathrm{D}$ triangular lattice, while the minimum value of the hole hopping term is only $-3 t$. So the lower edge of the extended state continuum is at $E_{-}=-9 t$ instead of $-2 z t=-12 t$. (Here $\mathrm{z}=6$ is the number of the nearest neighbors.) The eigenenergy of a single pair of hole and doublon on a triangular lattice is still given by Eq. (5). Now

$$
\epsilon_{\vec{k}}=-2 t\left[\cos k_{1}+\cos _{2}+\cos \left(k_{1}-k_{2}\right)\right]
$$

Here we have chosen the two primitive axes of the triangular lattice as the axes of our coordinate system. $k_{1}$ and $k_{2}$ are the components of $\vec{k}$ in the non-orthogonal basis which generates the reciprocal lattice. The minimum of $E_{\vec{K}}$ is reached when the total momentum of the hole-doublon pair $\vec{K}$ is at one of the corners of the hexagonal Brillouin zone, for example, $\vec{K}=(2 \pi / 3,-2 \pi / 3)$ in the non-orthogonal basis. Then Eq. (5) becomes

$$
A_{c} \int \frac{d^{2} q}{(2 \pi)^{2}} \frac{1}{E+2 \sqrt{3} t\left[\sin \left(\frac{\pi}{3}-q_{1}\right)+\sin \left(\frac{\pi}{3}+q_{2}\right)+\sin \left(\frac{2 \pi}{3}-q_{1}+q_{2}\right)\right]}=-\frac{1}{U} .
$$

Here $A_{c}=\sqrt{3} / 2$ is the area of the primitive cell and the integral is over the first Brillouin zone. The integral of Eq. (9) is logarithmically divergent as E approaches the lower edge of the extended state continuum $E_{-}=-9 t$ from below, which means that, just as on the square lattice, a single pair of hole and doublon on the nonbipartite triangular lattice also always forms a bound state no matter how small the interaction U is. Similarly, we can show that the conclusion is also valid for the $2 \mathrm{D}$ Hubbard model with the nearest and the next nearest neighbor hopping. In this case, the noninteracting energy dispersion on a square lattice is given by

$$
\epsilon_{k}=-2 t\left[\cos k_{x}+\cos k_{x}\right]-4 t_{2} \cos k_{x} \cos k_{y}
$$


Here the first and the second term correspond to the nearest neighbor and the next nearest neighbor hopping, respectively. For $t>2 t_{2}>0$, the minimum of $E_{K}$ is at $\vec{K}=(\pi, \pi)$, which is the same as the $t_{2}=0$ case. We can see that the question of whether a pair of hole and doublon always form a bound state for any finite $\mathrm{U}$ is only determined by the dimensionality of the system. For $d>2$, there exists a critical $U_{b}$, when and only when $U>U_{b}$, doublons and holes form charge neutral bound states; While for $d \leq 2$, they always form bound states for any finite $\mathrm{U}$. This conclusion is easily proved by considering the behavior of the integral in Eq.(5) near the point in k-space where $\epsilon_{\vec{k}}-\epsilon_{\vec{K}-\vec{k}}$ takes on its minimum value $E_{-}$. This is a variant of standard arguments about bound states. Its interest here is that two dimension is more similar to one dimension than it is to three dimensions in this limit of the Hubbard model, namely for high total spin but for all $\mathrm{U}$. This is contrary to the zero total spin case, where it is usually felt that one dimension is very special as regards the metal-insulator transition.

The conclusion that a bound state exists for all $\mathrm{U}$ in one and two dimensions and above a critical $U$ in three dimension is independent of lattice structures. This is well known in single particle quantum mechanics. We have simply reinterpreted the result as a metal-insulator transition at high $S_{z}$. The critical dimension for this transition is two in this model.

To verify that the bound state is indeed an insulating state, we follow Kohn [15] and calculate the charge stiffness constant $D_{c}$ which measures the response of the system to an electromagnetic field. We assume periodic boundary conditions along the x-direction and thread the system with a flux $\Phi$, which we represent by a vector potential $\vec{A}=\left(\Phi / N_{x}\right) \hat{e_{x}}$, where $N_{x}$ is the number of sites in the x-direction. The charge stiffness constant $D_{c}=$ $N_{x}^{2} d^{2} E(\Phi) / d \Phi^{2}$ is a measure of how good a conductor the system is, and vanishes for an insulator. 15, 16,20 Here $E(\Phi)$, the ground state energy in the presence of the flux $\Phi$, is given by,

$$
\frac{1}{N} \sum_{\vec{q}} \frac{1}{E(\Phi)-\epsilon_{\vec{q}}(\Phi)+\epsilon_{\vec{K}-\vec{q}}(-\Phi)}=-\frac{1}{U} .
$$

Here $\epsilon_{\vec{q}}(\Phi)$ is the single particle kinetic energy in the presence of the flux $\Phi$. For a 2D square 
lattice with the nearest neighbor hopping, for example, $\epsilon_{\vec{q}}(\Phi)=-2 t\left[\cos \left(q_{x}+\Phi / N_{x}\right)+\cos \left(q_{y}\right)\right]$. One can easily write down similar expressions for other cases. We expand Eq. (11) up to the second order of $\Phi$. The nth order equation gives $d^{n} E / d \Phi^{n}$ at $\Phi=0$. After some algebra, one finds that $d E / d \Phi$ is always zero, and $d^{2} E / d \Phi^{2}$ is zero when and only when $E<E_{-}$, i.e., $D_{c}=0$ when and only when there exists a bound state below the extended state band. This is because that only when $E<E_{-}=-8 t$, one can expand Eq. (11) in terms of $\Phi /\left[E+4 t\left(\cos q_{x}+\cos q_{y}\right)\right] ;$ When $E \geq E_{-}$, this expansion is not valid in some regions of the momentum space and $D_{c}$ is finite. This conclusion also applies to the $3 \mathrm{D}$ cubic lattice and other cases. We have therefore established the one-to-one correspondence between the existence of the bound state and the insulating behavior of the system. The bound state wavefunction contains precisely the hole-doublon phase coherence necessary for insulating behavior. This coherence can not be achieved when hole-doublon binding is accomplished by Gutzwiller-type projection methods. [16]

Next we consider an anisotropic three-dimensional system with small interlayer hopping. This is important in the study of high- $T_{c}$ superconductors. As we discussed above, the dimensionality of the system plays a very important role in determining whether the system is a Mott insulator or a metal. We can expect that even a small interlayer hopping could be quite important because it introduces the third dimension. Mathematically, the interlayer hopping term provides a cutoff to the 2D logarithmic divergence of Eq. (5). In this case, Eq. (5) can be written as

$$
\int \frac{d^{3} q}{(2 \pi)^{3}} \frac{1}{E+4 t\left(\cos q_{x}+\cos q_{y}\right)+4 t^{\prime} \cos q_{z}}=-\frac{1}{U} .
$$

Here $4 t^{\prime} \cos q_{z}$ is the interlayer hopping term. As before for $t^{\prime} \neq 0$, there exists a $U_{b}\left(t^{\prime}\right)$ such that when and only when $U>U_{b}\left(t^{\prime}\right)$, Eq. (11) has a bound state solution. In Fig. 1, we show the critical interaction $U_{b}\left(t^{\prime}\right)$ as a function of $t^{\prime}$. We can see that $U_{b}$ does depend on $t^{\prime}$ quite strongly in the small $t^{\prime}$ region. For example, at $t^{\prime}=0.001 t$, we still have $U_{b}=2.434 t$ even though $U_{b}=0$ at $t^{\prime}=0$. The crossover to $3 \mathrm{D}$ behavior occurs very rapidly as $t^{\prime}$ is turned on. 
When there are few doublons and holes, i.e., in the dilute limit, the overlap or interaction between the hole-doublon bound states (HDBS) is small and negligible, and the single pair HDBS solution gives a correct physical picture of the system. In $1 \mathrm{D}$ and 2D, the Hubbard model always has a charge gap at half-filling at high $S_{z}$, and therefore, is always an insulator for any finite $\mathrm{U}$ in the high- $S_{z}$ regime. (At least at $S_{z}=N / 2$, which is obvious, and at $S_{z}=N / 2-1$, which we have just proved rigorously). In $3 \mathrm{D}$, when the interaction $\mathrm{U}$ is smaller than $U_{b}$, there is no bound state and the system is a paramagnetic metal (or semimetal since the carrier density is small); when $U$ is larger than $U_{b}$, the doublons and holes form charge neutral bound states and the ground state is a gas of bound doublon-hole pairs, a very appealing model for an insulator. [1, 15, 16]

As the number of HDBS increases, the bound states merge into an energy band which corresponds to the lower Hubbard band in the original electron representation. Similarly, the extended state continuum corresponds to the upper Hubbard band, and the gap between the bound state and the extended state is nothing but the Mott-Hubbard gap. In 2D, we have shown that a single pair of hole and doublon always form a bound state and has a gap no matter how small the interaction $U$ is. The question is whether this gap will survive the overlap and the interaction between the HDBS pairs. That is the question we shall address in the next section.

\section{GENERAL TOTAL SPIN}

In this section we consider the Hubbard model at half-filling with general $S_{z}=(N-$ $2 M) / 2$, i.e., there are $\mathrm{M}$ spin-down electrons (or M doublon-hole pairs in HDR) in the halffilling N-site system. We exploit the fact that the relationship between the high spin limit and the lower spin case is very similar to that between the Cooper pairing problem and the BCS many-body problem. It is therefore natural for us to choose a BCS type of many-body wavefunction for the many-pair state. [14, 17] 


$$
\left|\vec{K}, S_{z}>=\prod_{k}\left(u_{k}+v_{k} d_{k}^{\dagger} h_{K-k}^{\dagger}\right)\right| 0>
$$

where $u_{k}^{2}=\frac{1}{2}\left(1+\xi_{k} / E_{k}\right), v_{k}^{2}=\frac{1}{2}\left(1-\xi_{k} / E_{k}\right), E_{k}^{2}=4 \Delta^{2}+\xi_{k}^{2}$, and $\xi_{k}=\epsilon_{k}-\epsilon_{K-k}-\mu_{d}-\mu_{h}$ is the kinetic energy of the Cooper pair measured from the Fermi surface. $\mu_{d}$ and $\mu_{h}$ are the chemical potential of the doublons and the holes, respectively, and $\Delta=\frac{U}{N} \sum_{\vec{k}} u_{k} v_{k}$ is the BCS gap function which is momentum-independent in this case because of the short-range interaction in Hubbard model. $\vec{K}$ is the total momentum of the hole-doublon "Cooper pairs" which can also be thought as the wavevector of the spin-density wave in the original electronic picture. [9,17] This kind of pairing state was first studied by Fulde and Ferrell, and Larkin and Ovchinnikov in the context of superconductivity. [17,18 Obviously this wavefunction is not an eigenfunction of operator $\hat{S}_{z}$, and the labeling $S_{z}$ on the left-hand side of Eq.(13) should be understood in the sense of the average expectation value of operator $\hat{S}_{z}$ just as the number of particles in the BCS case. Usually $\vec{K}$ is at one of the corners of the first Brillouin zone as we can see from the single-pair solution. It could move away from the corners in some cases, which corresponds to the incommensurate spin-density wave.

We evaluate the expectation value of the Hamiltonian Eq. (2) using the wavefunction of (13). The gap equation is then given by minimizing the energy expectation value [19]

$$
\frac{1}{N} \sum_{\vec{k}} \frac{1}{\left[\left(\epsilon_{k}-\epsilon_{K-k}-2 \mu\right)^{2}+4 \Delta^{2}\right]^{1 / 2}}=\frac{1}{U}
$$

Here $2 \mu=\mu_{d}+\mu_{h}$. The total z spin is given by $S_{z}=N / 2-\sum_{k} v_{k}^{2}$, which, together with the gap equation, can be used to calculate the chemical potential $\mu$ and the gap $\Delta$,

$$
\frac{1}{N} \sum_{\vec{k}}\left[1-\frac{\epsilon_{k}-\epsilon_{K-k}-2 \mu}{\left[\left(\epsilon_{k}-\epsilon_{K-k}-2 \mu\right)^{2}+4 \Delta^{2}\right]^{1 / 2}}\right]=1-\frac{2 S_{z}}{N} .
$$

Here $\mathrm{N}$ is the number of sites. It is easy to demonstrate that in $2 \mathrm{D}$, Eq.(14) always has a nonzero solution $\Delta$ for any finite $\mathrm{U}$, which means that there is always a charge excitation gap in this particular wavefunction for any finite value of $\mathrm{U}$. To show that, we take the limit $N \rightarrow \infty$ and replace the sum $\sum_{\vec{k}}$ with the integral over the first Brillouin zone,

$$
A_{c} \int \frac{d^{2} k}{(2 \pi)^{2}} \frac{1}{\left[\left(\epsilon_{k}-\epsilon_{K-k}-2 \mu\right)^{2}+4 \Delta^{2}\right]^{1 / 2}}=\frac{1}{U} .
$$


Here $A_{c}$ is the area of the primitive unit cell. $A_{c}=1$ for the square lattice and $A_{c}=\sqrt{3} / 2$ for the triangular lattice. The integral in Eq. (16) is logarithmically divergent as $\Delta$ goes to zero. This is due to the fact that generally in $d=2,\left(\epsilon_{\vec{k}}-\epsilon_{K_{-k}}-2 \mu\right)$ vanishes along a curve in the momentum space. That is sufficient to cause the logarithmic divergence of the left-hand-side of Eq. (16) as $\Delta \rightarrow 0$.

This reasoning would suggest that the 2D Hubbard model is an insulator at half filling for any finite $\mathrm{U}$, as is true for the 1D model, and the square lattice with the nearest neighbor hopping only. To understand how the reasoning breaks down, we consider three cases:

(A). bipartite square lattice with the nearest neighbor (NN) hopping only,

(B). square lattice with NN and the next nearest neighbor (NNN) hopping, and

(C). nonbipartite triangular lattice with nearest neighbor hopping only.

\section{A. Square lattice with NN hopping only}

In this case, $\epsilon_{\vec{K}-\vec{k}}=-\epsilon_{\vec{k}}$, and the many-body wavefunction (113) at $\Delta=0$ is identical to the noninteracting wavefunction. The fact that the gap equation always has a nonzero solution for any finite $\mathrm{U}$ means that the Fermi liquid state is unstable against the BCS state (13) at finite $\mathrm{U}$. The staggered magnetization of Hubbard model is proportional to $\Delta / U$. So obviously a nonzero $\Delta$ means that the system is antiferromagnetically ordered. We therefore conclude that the system is always an antiferromagnetic insulator for any finite U. This result is well known, but the present method gives a new way of visualizing it. Consider the first Brillouin zone in Fig.2(a). The doublons occupy the region near $\vec{K}=0$, and the holes occupy the region near $\vec{K}=(\pi, \pi)$. The energy curves are exact translates of one another through $(\pi, \pi)$ for all fillings, so each hole pairs with one doublon. This particular model is known to have perfect nesting at half filling, but note that this property is even stronger. There is a perfect particle-hole nesting at all values of the magnetization. As a result, the pairing state is always energetically favorable.

In the large $\mathrm{U}$ limit, one can solve the gap equation (14) by expanding the gap function $\Delta$ in terms of $t / U$. At $S_{z}=0$, we find 


$$
\Delta=\frac{1}{2} U\left[1-8 \frac{t^{2}}{U^{2}}+O\left(\frac{t^{4}}{U^{4}}\right)\right]
$$

and the expectation value of the Hamiltonian in the pairing state is

$$
E_{0}=<\vec{K}, S_{z}=0|H| \vec{K}, S_{z}=0>=-4 N t\left[\frac{t}{U}+O\left(\frac{t^{3}}{U^{3}}\right)\right]
$$

which is the expected result $-N J$ for a Néel state, and shows that our mean-field solution has the correct large-U limit. Here $J=4 t^{2} / U$. The pairing state (13) may do better when $\mathrm{U}$ is small. The reason is that in this case the size of bound states becomes large, there are many particles inside the region covered by a single bound state wavefunction, which will suppress the relative fluctuation in the interaction effects, and the BCS mean-field wavefunction becomes a better approximation to the exact ground state.

\section{B. Square lattice with NN and NNN hopping}

The noninteracting energy dispersion in this case is given by Eq. (10). The crucial difference between the case (A) and (B) is that for case (B), $\epsilon_{K-k} \neq-\epsilon_{k}$, and the BCS manybody wavefunction (13) does not reduce to the noninteracting wavefunction when $\Delta=0$, i.e., the fact that the gap equation (14) always has a nonzero solution for any finite $\mathrm{U}$ just means that the wavefunction (13) at $\Delta=0$ is always unstable against the finite- $\Delta$ BCS pairing state, but the $\Delta=0$ wavefunction is not necessarily the Fermi liquid wavefunction. Thus in HDR, when the holes and doublons take advantage of the attractive interaction between them and form the BCS condensate, they pay the price of having a little higher kinetic energy in case (B). This can be seen in Fig. 2(b), where the doublon Fermi surface near $\mathbf{K}=(0,0)$ can not be mapped onto the hole Fermi surface near $\mathbf{K}=(\pi, \pi)$ through a linear transformation $\mathbf{k}^{\prime}=\mathbf{K}-\mathbf{k}$. It is easy to show that the Fermi liquid state has lower kinetic energy than the pairing state (13). This problem becomes severe at low $S_{z}$. So when the gain from the condensation energy is not enough to compensate the extra kinetic energy, the BCS state (13) becomes unstable to the Fermi liquid state, the system goes through a first order phase transition and becomes a paramagnetic metal (Fermi liquid). Once we

have established the correct physical picture, it becomes straightforward to calculate the 
metal-insulator phase boundary on which the energy of the many-body wavefunction (13) is equal to the energy of a true Fermi liquid state. In Fig. 3, we show the calculated phase diagram of the case (B) with $t_{2}=0.25 t$ in the $S_{z}$-U plane. For $S_{z}=0$, the critical interaction $U_{c}$ is about 2.3t, which is in agreement with the result of the numerical and Hartree-Fock calculation by Lin and Hirsch. [4] As $t_{2}$ goes to zero, case (B) reduces to case (A), and the critical interaction of the metal-insulator transition $U_{c}$ goes to zero.

\section{Triangular lattice with NN hopping}

Case (C) is more complicated than case (B), due to the conflict between the "pairing" property of the wavefunction (13) and the "tripartite" character of the lattice. As a result, the holes of the wavefunction (13) only occupy three of the six corners of the hexagonal first Brillouin zone (BZ), leaving the low energy states of the other three corners unoccupied or partially occupied. The occupied three corners, as well as the unoccupied three corners, are related by the reciprocal lattice vectors and form two subsets, respectively, but these two subsets are not related by the reciprocal lattice vectors. As shown in Fig.4(a), the hexagonal BZ (dotted line) is equivalent to the parallelogram (solid line) which is easier to deal with analytically. So by choosing $\mathbf{K}$ to be at one of the BZ corners (or equivalently, two points $\mathrm{A}$ and $\mathrm{B}$ on the diagonal of the parallelogram which divided the diagonal into three equal segments), we force the system breaking the point group symmetry as well as the time reversal symmetry. In Fig. 4(a), we also show the Fermi surfaces of the doublons (solid line) and holes (dashed line) at $S_{z}=0.4 N$ and $0.3 N$, respectively. Apparently there are two minimum energy positions (which correspond to two distinguish sets of corners of the hexagonal BZ) for holes to occupy, but in the pairing state (13) they can only occupy one of them and leave the other unoccupied. This, as well as the distortion of the Fermi surface, costs extra kinetic energy, which opens doors for those many-body states other than the Fermi-liquid or the state of (13) with $\mathbf{K}$ at the one of the corners of BZ as the possible candidate of the ground state in some parameter region. One possible candidate is the incommensurate spin-density wave state which corresponds to a Fulde-Ferrell-LarkinOvchinnikov (FFLO) type of state in HDR. In comparison, the four corners of the BZ of the 
square lattice are related by the reciprocal lattice vector and the increasing of the kinetic energy in the pairing state in the NNN hoping case is solely due to the distortion of the Fermi surface. For this reason, we expect the critical interaction $U_{c}$ of the instability of the BCS state (13) in case (C) to be larger than that in case (B), and the system may go through other kind of states before becoming a paramagnetic metal (Fermi liquid).

We calculate the energy of the pairing state (13) for general $\mathbf{K}$. We find that the energy of the pairing states $\mathbf{K}$ at the corner of first Brillouin zone is at least a local minimum. It is quite possibly also a global minimum. Since there are two set of the optimum $\mathbf{K}$ which are not related to each other by the reciprocal lattice vectors, the triangular lattice has two degenerate ground states which may have some interesting consequences. For example, the system may be vulnerable to the Jahn-Teller distortion.

In Fig. 4(b), we show the critical interaction $U_{c}$ of the Hubbard model on a triangular lattice as a function of total spin $S_{z}$. Below $U_{c}$, the BCS state (13) is unstable against the paramagnetic Fermi liquid state. We find, as expected, that $U_{c}$ is larger than the square lattice with NNN hoping case due to the incompatibility of "pairing" and the tripartite nature of the lattice. Our calculated critical interaction of the 2D Hubbard model on a triangular lattice $U_{c}=4.936 t$ at $S_{z}=0$ is a little bit smaller than the result of Krishnamurthy et. al. [9,20], their metal-insulator transition is at $U_{c 2}=5.27 t$. But our result is not in contradiction with their result, because Krishnamurthy et al. have considered a more complicated metallic state, namely the spin density wave metallic state while we only compare our pairing state with the most simple Fermi liquid state. So our result shown in Fig. 4(b) is the lower bound of the critical interaction of the metal-insulator transition.

\section{AWAY FROM HALF-FILLING}

In section II and III, we discussed the properties of Hubbard model on various lattices at half-filling. In HDR, this corresponds to the case where the number of doublons is equal to the number of holes. In this section we consider the case of Hubbard model away from 
the half-filling. One natural choice for the wavefunction of the hole-doped system is

$$
\left|\psi_{n}>=\sum_{\left\{q_{i}\right\}} A\left(\left\{q_{i}\right\}\right) \prod_{i=1}^{n} \gamma_{q_{i}}^{\dagger}\right| \psi_{0}>
$$

where $\gamma_{p}^{\dagger}=u_{p} h_{K-p}^{\dagger}+v_{p} d_{p}$ is the creation operator of one of the Bogoliubov quasiparticle species, 19,21 $\left|\psi_{0}>\equiv\right| \mathbf{K}, S_{z}>$ is the pairing wavefunction for the half-filled case (cf. Eq. (13)). $n=N_{h}-N_{d}, N_{h}$ and $N_{d}$ is the number of holes and doublons, respectively. The wavefunction (19) should remain reasonably good for the multi-hole-doped system as long as it still has the long-range order. Especially, the wavefunction of the system with one extra-hole $(n=1)$ is

$$
\left|\psi_{1}>=\sum_{q} A_{q} \gamma_{q}^{\dagger}\right| \psi_{0}>
$$

which represents a series of states from the Nagaoka state to the antiferromagnetic state with one extra-hole as one moves from high- $S_{z}$ to $S_{z}=0$. We evaluate the expectation value of $H$ in the state $(20)$,

$$
<\psi_{1}|H| \psi_{1}>=\sum_{\vec{k}}\left(\epsilon_{k}-\epsilon_{K-k}\right) v_{k}^{2}-N \frac{\Delta^{2}}{U}+N_{d} U\left(1-\frac{N_{d}}{N}\right)+E_{1}\left(\left\{A_{k}\right\}\right) .
$$

Here $E_{1}\left(\left\{A_{k}\right\}\right)=<\psi_{1}|H| \psi_{1}>-<\psi_{0}|H| \psi_{0}>$ is the energy of the extra-doped hole,

$$
E_{1}\left(\left\{A_{k}\right\}\right)=\sum_{\vec{k}}\left[2 \Delta u_{k} v_{k}-U\left(1-2 \frac{N_{d}}{N}\right) v_{k}^{2}-\epsilon_{k} v_{k}^{2}-\epsilon_{K-k} u_{k}^{2}\right]\left|A_{k}\right|^{2}-U \frac{N_{d}}{N}
$$

From that we can find the optimum $\left\{A_{k}\right\}$ which minimizes $E_{1}$ with the constraint $\sum_{\vec{k}}\left|A_{k}\right|^{2}=$ 1 as well as the bandwidth of the doped hole which is the difference between the maximum and the minimum of $E_{1}$. For simplicity, we consider the 2D Hubbard model on a square lattice with the nearest neighbor hopping only. We find that the optimum $A_{k}=\delta\left(\epsilon_{k}-\mu-\eta\right)$ and the minimum energy of the doped hole is

$$
E_{\min }=\sqrt{\eta^{2}+\Delta^{2}}-U / 2+\left(\mu+\frac{U}{2}-U \frac{N_{d}}{N}\right) \eta / \sqrt{\eta^{2}+\Delta^{2}}
$$

Here $\eta$ is the optimum $\left(\epsilon_{k}-\mu\right)$ which minimizes the hole energy. It is given by

$$
\eta^{3}+\eta \Delta^{2}+\left(\mu+\frac{U}{2}-U \frac{N_{d}}{N}\right) \Delta^{2}=0,
$$


with the constraint

$$
-4 t-\mu \leq \eta \leq 4 t-\mu
$$

When $S_{z}$ is zero, $N_{d} / N=1 / 2, \mu=0$, therefore $\eta=0$, i.e., the hole is right on the fermi surface, and

$$
E_{\min }=\Delta-U / 2 \text {. }
$$

Here $\Delta$ is the solution of the gap equation (14). In Fig. 5(a), we show the minimum hole energy $E_{\min }$ as a function of $\mathrm{U} / \mathrm{t}$ for a square lattice with the nearest-neighbor hopping only. One can see that in the small $U$ region, $E_{\text {min }}$ decreases as $U$ increases. It reaches a minimum at $U / t \approx 2.7$. In the large $U$ limit, $\Delta$ can be written down explicitly as a Taylor series of $t / U$ (cf. Eq. (17)). We get

$$
E_{\text {min }}=-\frac{4 t^{2}}{U}+O\left(\frac{t^{4}}{U^{3}}\right)=-J+O\left(\frac{t^{4}}{U^{3}}\right)
$$

Similarly, we find that the maximum of the hole energy at $S_{z}=0$ is,

$$
E_{\text {max }}=\sqrt{(4 t)^{2}+\Delta^{2}}-U / 2
$$

In the large U limit,

$$
E_{\max }=12 \frac{t^{2}}{U}+O\left(\frac{t^{4}}{U^{3}}\right)=3 J+O\left(\frac{t^{4}}{U^{3}}\right)
$$

and the bandwidth of the doped hole,

$$
W=4 J+O\left(\frac{t^{4}}{U^{3}}\right)
$$

This is in agreement with the recent numerical calculations which find that a hole in an antiferromagnetic background has a bandwidth in the scale of $J$ rather than $t$, in the small $J / t$ limit. 22 26

In Fig. 5(b), we plot the bandwidth

$$
W=E_{\max }-E_{\min }=\sqrt{(4 t)^{2}+\Delta^{2}}-\Delta
$$


as a function of $\mathrm{U}$ at $S_{z}=0$. We can see that $\mathrm{W}$ is proportional to $\mathrm{t}$ in the small $\mathrm{U}$ region where $\Delta \ll t$. It smoothly crosses over to scale as $\mathrm{J}$ in the large $\mathrm{U}$ region where $\Delta \gg t$. Physically, this is in agreement with the string picture of a hole in an antiferromagnetic environment. 24,27] Due to the presence of the frustrated spin string left behind the hopping hole, the hole acquires a large effective mass $m^{*}$ which is proportional to the inverse of the effective hopping $t^{*}$, and therefore, also to the inverse of the bandwidth $\mathrm{W}$. Due to the presence of the interaction, $t^{*}$ is renormalized from t to $J=4 t^{2} / U$ in the large $\mathrm{U}$ limit.

When $S_{z} \neq 0$, the chemical potential $\mu$ is not zero anymore and is given by Eq. (15). In Fig. 6 we show the minimum hole energy as a function of $S_{z}$ for $U / t=32,16$, and 8, respectively. We can see that $E_{\text {min }}$ decreases as one increases $S_{z}$ from zero, i.e., it costs less to dope a hole in the high- $S_{z}$ states than in the lower $S_{z}$ states. So the doping of holes suppresses the gap and may stabilize the high $S_{z}$ state. When the decrease of the hole energy becomes larger than the energy difference between the ferromagnetic and the antiferromagnetic configurations in the undoped case, the system goes through a phase transition from the antiferromagnetism to the Nagaoka ferromagnetism. In the large U limit, $\mu$ and $\Delta$ can be calculated analytically by Taylor expanding Eqs. (14) and (15),

$$
\begin{aligned}
& \mu=\left(-1 / 2+N_{d} / N\right) U+O\left(t^{2} / U\right), \\
& \Delta=\left[\frac{N_{d}}{N}\left(1-\frac{N_{d}}{N}\right)\right]^{1 / 2} U+O\left(t^{2} / U\right) .
\end{aligned}
$$

Here $N_{d}$ and $N$ is the number of doublons and lattice sites, respectively, $S_{z}=N / 2-N_{d}$. The $S_{z} \neq 0$ case can again be divided into two cases due to the constraint Eq. (25). First, when $4 t \geq\left(1 / 2-N_{d} / N\right) U$, from Eqs. (24) and (32) we get $\eta=a t^{2} / U$ in the large U limit, here $a$ is a number of order of 1 . The minimum hole energy

$$
E_{\min }=\Delta-U / 2+O\left(\frac{t^{4}}{U^{3}}\right)
$$

As $S_{z}$ increases from zero, $\Delta$ decreases since the number of the doublon-hole pairs is equal to $\left(N / 2-S_{z}\right)$. Therefore $E_{\text {min }}$ decrease as $S_{z}$ increases from zero. Second, when $4 t<$ 
$\left(1 / 2-N_{d} / N\right) U$, the optimum $\left(\epsilon_{k}-\mu\right)$ one can get is $\eta=\left(1 / 2-N_{d} / N\right) U-4 t$ due to the constraint Eq. (25), and

$$
E_{\text {min }}=\sqrt{\eta^{2}+\Delta^{2}}-U / 2
$$

in the large $\mathrm{U}$ limit. Again, $E_{\min }$ can be written as a Taylor series,

$$
E_{\min }\left(S_{z}\right)=-4 t\left(1-\frac{2 N_{d}}{N}\right)+O\left(t^{2} / U\right) .
$$

Finally, we have also calculated the energy of two doped holes,

$$
E_{2}=<\psi_{2}|H| \psi_{2}>-<\psi_{0}|H| \psi_{0}>
$$

using the variational wavefunction,

$$
\left|\psi_{2}>=\sum_{k, q} A_{k, q} \gamma_{k}^{\dagger} \gamma_{q}^{\dagger}\right| \psi_{0}>
$$

Here $A_{k, q}$ is determined by minimizing $E_{2}$. The quantity $E_{2}-2 E_{1}$ gives information about the interaction between two holes. We find

$$
E_{2}-2 E_{1}=\frac{4 U}{N} \sum_{k_{1}, k_{2}, k_{3}} v_{k_{1}} v_{k_{2}} u_{k_{3}} u_{k_{2}+k_{3}-k_{1}} A_{k_{1}, k_{2}+k_{3}-k_{1}} A_{k_{2}, k_{3}}^{*},
$$

which goes to zero in the thermodynamic limit. This is because we did not allow the gap function $\Delta$ to fluctuate in our calculation. It is believed that the fluctuation of the antiferromagnetic order parameter will result in an attractive interaction between holes, and this attractive interaction favors a $d_{x^{2}-y^{2}}$ type of pairing. 28]

To summarize the results of Sec. IV, we have extended the pairing wavefunction for the repulsive Hubbard model to the case of less than half-filling by using the Bogoliubov quasi-particle operators. We use this variational wavefunction to calculate the energy of a doped hole. We find that in the large $\mathrm{U}$ limit, the doped hole has a bandwidth of order of J rather than t at $S_{z}=0$. 229] Here $J=4 t^{2} / U$ is the magnetic interaction strength. The hole energy decreases as one increases $S_{z}$. The competition between this effect and the Heisenberg spin interaction which favors antiferromagnetism determines the transition 
between the antiferromagnetic state and the Nagaoka ferromagnetic state. Our variational wavefunction has the advantage of capable of representing a series of states in between these two limits in a single, relatively simple form.

It also possible to view the system in the limit of high spin near half filling as a low density, two component system. Field theory methods can be applied in this limit. This approach lies outside the variational method of this paper, but will be described in a future publication.

\section{CONCLUSIONS}

In conclusion, we have studied the metal-insulator transition and magnetic ordering in half-filled Hubbard model using a particle-hole mapping. We show that the 2D Hubbard model on any lattice is an insulator at half-filling for any finite $\mathrm{U}$ in the (Nagaoka) high spin region. The physical picture of this metal-insulator transition as a function of spin is quite simple from the point of view of binding and unbinding of the hole-doublon pairs. At high spin $S_{z}$, the low energy excitations are neutral, consisting of the motion of hole-doublon pairs. In this region, the dimensionality plays a very important role in determining whether the system is a metal or a Mott insulator. For $d>2$, there exists a critical $U_{b}$, when and

only when $U>U_{b}$, doublons and holes form charge neutral bound states and the system is a Mott insulator in the high spin region; while for $d \leq 2$, holes and doublons always form bound states and the system is an insulator for any finite $U$ in the high spin region. As one increases the number of bound states, i.e., lowers $S_{z}$, the binding costs some extra kinetic energy if the Fermi surface is not nested. When the cost of kinetic energy exceeds the binding energy, a binding-unbinding transition takes place. The excitations are charged, and the system is a metal. If the Fermi surface is nested, however, this binding costs no extra kinetic energy, and the system is always an insulator for any finite $\mathrm{U}$ at any spin $S_{z}$. We have also discussed the generalization of the pairing wavefunction (13) to the hole doped case using Bogoliubov quasiparticle operators. We find that the dispersion of the doped 
hole is significantly changed due to the presence of the interaction. In the large U limit, the doped hole has a bandwidth of order of J rather than t at $S_{z}=0$.

\section{ACKNOWLEDGMENTS}

It is a pleasure to thank Elbio Dagotto, Richard Ferrell, Steve Girvin, Adriana Moreo, Mohit Randeria, and Nandini Trivedi for valuable discussions. We would also like to thank the hospitality of the Aspen Center for Physics where part of the work was done. This work was supported by the NSF through Grant No. DMR 9214739, and by the Electric Power Research Institute. We also acknowledge support from San Diego Supercomputer Center. 


\section{REFERENCES}

[1] J. Hubbard, Proc. Roy. Soc. London A 276, 238 (1963); 281, 401 (1964).

[2] E. Fradkin, Field Theories of condensed matter systems, (Addison-Wesley, Redwood City, California, 1991).

[3] N. F. Mott, Metal Insulator Transitions, (Taylor and Francis, London, 1974).

[4] J. E. Hirsch, Phys. Rev. B 31, 4403 (1985); H. Q. Lin and J. E. Hirsch, Phys. Rev. B 35, 3359 (1987).

[5] Y. Nagaoka, Phys. Rev. 147, 392 (1966).

[6] P. W. Anderson, Solid State Phys. 14, 99 (1963).

[7] P. W. Anderson, Science 235, 1196 (1987); F. C. Zhang and T. M. Rice, Phys. Rev. B 37, 3759 (1988).

[8] G. Kemeny and L. G. Caron, Rev. Mod. Phys. 40, 790 (1968); Y. Nagaoka, Prog. Theor. Phys. 52, 1716 (1974); V. J. Emery, Phys. Rev. B 14, 2989 (1976); R. R. P. Singh and R. T. Scalettar, Phys. Rev. Lett. 66, 3203 (1991).

[9] H. R. Krishnamurthy, C. Jayaprakash, S. Sarker, and W. Wenzel, Phys. Rev. Lett. 64, $950(1990)$.

[10] Q. P. Li and R. Joynt, Phys. Rev. B 47, 3979 (1993).

[11] A. J. Leggett, in Modern Trends in the Theory of Condensed Matter, edited by A. Pekalski and R. Przystawa (Springer, Berlin, 1980).

[12] P. Nozieres and S. Schmitt-Rink, J. Low Temp. Phys. 59, 195 (1985).

[13] M. Randeria, J.-M. Duan, and L.-Y. Shieh, Phys. Rev. Lett. 62, 981 (1989); K. Miyake, Prog. Theor. Phys. 69, 1794 (1983).

[14] J. R. Schrieffer, Theory of Superconductivity, (Benjamin/Cummings, Reading, MA, 
1971).

[15] W. Kohn, Phys. Rev. 133, A171 (1964).

[16] A. J. Millis and S. N. Coppersmith, Phys. Rev. B 43, 13770 (1991).

[17] P. Fulde and R. A. Ferrell, Phys. Rev. 135, A550 (1964); C. N. Yang, Phys. Rev. Lett. 63, 2144 (1989).

[18] A. I. Larkin and Yu. N. Ovchinnikov, Zh. Eksp. Teor. Fiz. 47, 1136 (1964). [Sov. Phys. JETP 20, 762 (1965).]

[19] P. G. de Gennes, Superconductivity of Metals and Alloys, (Addison-Wesley, Redwood City, CA, 1989) Chapter 4.

[20] S. Sarker, C. Jayaprakash, H. R. Krishnamurthy, and W. Wenzel, Phys. Rev. B 43, 8775 (1991); C. Jayaprakash, H. R. Krishnamurthy, S. Sarker, and W. Wenzel, Europhys. Lett. 15, 625 (1991).

[21] N. N. Bogoliubov, Nuovo Cimento 7, 794 (1958); J. G. Valatin, Nuovo Cimento 7, 843 (1958).

[22] E. Dagotto, A. Moreo, R. Joynt, S. Bacci, and E. Gagliano, Phys. Rev. B 41, 2585 (1990).

[23] K. von Szczepanski, P. Horsch, W. Stephan, and M. Ziegler, Phys. Rev. B 41, 2017 (1990).

[24] S. Trugman, Phys. Rev. B 41, 892 (1990); 37, 1597 (1988).

[25] D. Poilblanc and E. Dagotto, Phys. Rev. B 44, 466 (1991).

[26] A. V. Chubukov and D. M. Frenkel, Phys. Rev. B 46, 11884 (1992).

[27] E. Dagotto, preprint.

[28] P. Monthoux, A. V. Balatsky, and D. Pines, Phys. Rev. Lett. 67, 3448 (1991); P. 
Monthoux and D. Pines, Phys. Rev. Lett. 69, 961 (1992).

[29] W. F. Brinkman and T. M. Rice, Phys. Rev. B 2, 1324 (1970). 


\section{FIGURES}

FIG. 1. Critical interaction $U_{b}$ as a function of the interlayer hopping constant $t^{\prime}$ in a $3 \mathrm{D}$ bipartite lattice. When $U>U_{b}$, a pair of hole and doublon forms charge neutral bound state and Hubbard model is an insulator at the high spin region.

FIG. 2. (a). Fermi surfaces of doublons (solid lines) and holes (dashed lines) of the 2D Hubbard model on a square lattice with the nearest neighbor hopping only at various $S_{z}$. (b). Fermi surfaces of doublons (solid lines) and holes (dashed lines) of the 2D Hubbard model on a square lattice with the nearest and the next nearest neighbor hopping at $S_{z}=0.4 N, 0.3 N$, and $0.2 N$. The parameter is $t_{2}=0.25 t$.

FIG. 3. Critical interaction $U_{c}$ of the 2D Hubbard model on a square lattice with $\mathrm{NN}$ and NNN hopping as a function of $S_{z}$. When $U>U_{c}$, holes and doublons form charge neutral bound states and the system is an insulator. The parameter is the same as in Fig. 2(b).

FIG. 4. (a) Fermi surfaces of doublons (solid lines) and holes (dashed lines) of the Hubbard model on a $2 \mathrm{D}$ triangular lattice with the nearest neighbor hopping only at various $S_{z}$. (b). Critical interaction $U_{c}$ of the Hubbard model on a triangular lattice with NN hopping as a function of $S_{z}$. When $U>U_{c}$, holes and doublons form charge neutral bound states and the system is an insulator.

FIG. 5. Shows the calculated (a) minimum hole energy, and (b) the bandwidth of a single doped hole of the 2D Hubbard model on a square lattice with the nearest neighbor hopping as a function of $U / t$ at $S_{z}=0$ and one-hole away from half-filling.

FIG. 6. Shows the minimum hole energy of 2D Hubbard model on a square lattice as a function of $S_{z}$ for $U=32 t$ (solid), $16 t$ (dashed), and $8 t$ (dotted), respectively. 